http://jmscr.igmpublication.org/home/ ISSN (e)-2347-176x ISSN (p) 2455-0450

crossref DOI: https://dx.doi.org/10.18535/jmscr/v8i2.56

\author{
Journal Of Medical Science And Clinical Research

\title{
Clinicopathological study of Carcinoma Breast
}

Authors

\author{
Dr Lalitha Kumari Dumpala ${ }^{1}$, Dr N. Jagadesh $\mathrm{MS}^{2}$, Dr Rohini $\mathrm{D}^{3}$, Dr Harsha \\ ${ }^{1,3,4}$ Post Graduate, Department of General Surgery, Andhra Medical College, Visakhapatnam \\ ${ }^{2}$ Assistant Professor, Department of General Surgery, Andhra Medical College, Visakhapatnam \\ *Corresponding Author
}

Dr Lalitha Kumari Dumpala

\begin{abstract}
Introduction: Breast cancer is the most common site-specific cancer in women and is the leading cause of death from cancer for women aged 20 to 59 yrs. It accounts for $27 \%$ of all newly diagnosed cancers in females and is responsible for $16 \%$ of the cancer- related deaths in women.

So a study based on clinical staging, pathological staging, and hormone receptor status is essential in the appropriate management of breast cancer patients.
\end{abstract}

Material and Methods: 50 breast cancer patients who attended the department of general surgery, king George hospital, Andhra medical college, Visakhapatnam between august 2017 to June 2019.

Result

- The commonest stages of presentation were stage II and stage III in both clinical and pathological staging.

- Patients with large tumors have advanced lymph node status

- There is between good linear correlation clinical stage and pathological stage

- $50 \%$ of cases have presented without lymphnodes

- Triple-negative cases were more common in patients with the advanced clinical and pathological stage. O Testing for ER, PR, Her2neu receptor status is recommended in all patients with breast cancer to facilitate neoadjuvant and adjuvant therapy and for assessing prognosis.

\section{Conclusion}

- Breast cancer is the most common site-specific cancer in women

- Carcinoma breast is mainly a disease of fifth and sixth decades. However, there is a rising incidence in younger population o Carcinoma breast most commonly affected right breast than left breast in my study with upper and outer quadrant being most commonly involved

- Young patients have presented with advanced-stage disease

Keywords: Breast cancer, Breast lump, Infiltrating ductal carcinoma.

\section{Introduction}

Carcinoma breast is the most common cancer in women and is the leading cause of death from cancer for women aged 20 to 59 years. It accounts for $27 \%$ of all newly diagnosed cancers in females and is responsible for $16 \%$ of cancer related deaths in women. So, a study based on clinical staging, pathological staging, and hormone receptor status is 
essential in appropriate management of breast cancer patients

\section{Aims and Objectives}

$>$ To study age distribution.

$>$ To study the distribution of the clinical stage.

$>$ To study the correlation between clinical and pathological staging.

To study the distribution of hormone receptor status.

\section{Materials and Methods}

50 breast cancer patients who attended the department of general surgery, king George hospital, Andhra medical college, Visakhapatnam between august 2017 to June 2019 .

Inclusion Criteria: All cases of carcinoma breast irrespective of age and sex. All cases of carcinoma breast irrespective of any stage.

Exclusion Criteria: Patients not willing to give consent.

\section{Results}

In study majority of the patients presenting with carcinoma breast are in the fifth and sixth decade (27f50), followed by third and fourth decade (18f50), and Only 5 Patients are in the age group of $61-80 y$ rs. Eighteen were $<40 y$ rs with a median age of $38 y r s, 32$ were $>40 y r s$ with a median age of $55 \mathrm{yrs}$. The youngest patient was $30 \mathrm{yrs}$ old. The oldest patient was $80 \mathrm{yrs}$ old.

\section{Age Distribution}

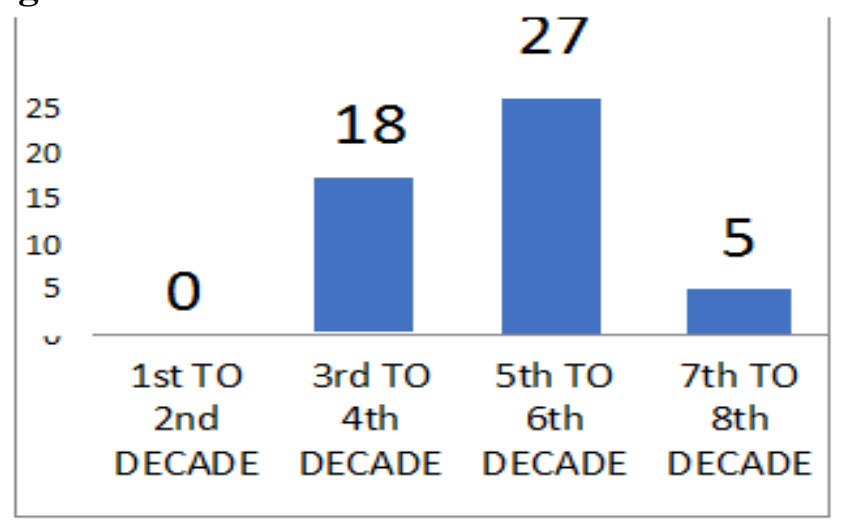

Sex Distribution It more common in females (49f50) than males ( $1 f 50)$.

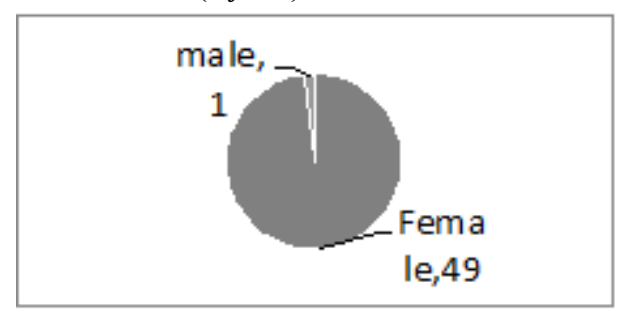

Site Distribution: In this study, the right breast (26f50) was more commonly affected than the left breast (23f50), and only 1 case was affected with bilateral breast carcinoma. The most common site involved in the upper and outer quadrant (36f50) followed by central quadrant ( $7 f 50)$, lower and outer quadrant (4f50), and upper inner quadrant (3f50).

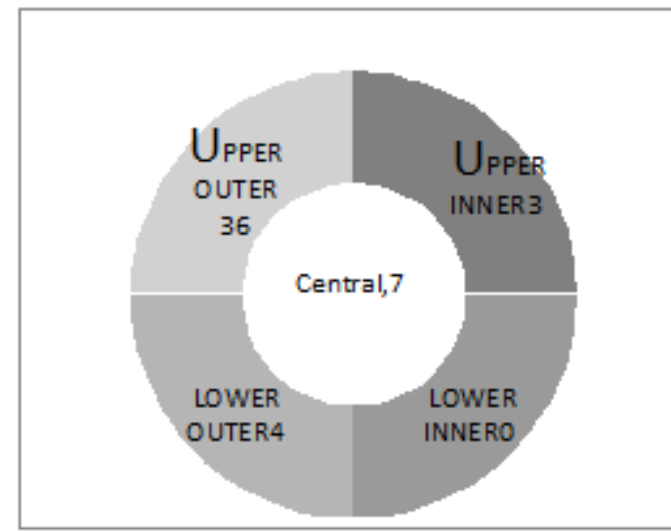

Clinical Staging: In this study, 24 patients were in stage II, and 26 patients were in stage III. Stage I and stage IV disease were not encountered during the period of this study. Of the 18 patients in the age group 21-40yrs, eight patients presented with stage II and ten patients presented with stage III. Of the 27 patients in the age group 41-60yrs, 13 patients presented with stage II, whereas 14 presented with stageIII.Ofthefivepatients'61-80yrs age group, three patients presented with stage II and two presented with stage III.

Patients of a young age have presented with advanced clinical stage. In the present study, the majority of 20 presented with T2 stage, 15 presented with T3, and remaining with T4. No patient with T1 was encountered in this study while studying the nodal status of the disease 20 presented with NO stage, 25 presented with N1 Stage, and five presented with $\mathrm{N} 2$ stage. 12 out of 20 patients with T2 tumors had NO status, 9 out of 15 patients with 
T3 tumors had N1 status, and 7 out of 15 patients with T4 tumors had N1 status. Two patients with T3 tumors and three patients with T4 tumors had N2 status. Patients with massive tumors have more advanced lymph nodestatus.

Pathological Staging: In this study (20f50), were in pathological stage II and (30f50) were in pathological stage III. Pathological Stage I and stage IV disease were not encountered during the period of the study. Of 18 patients in the age group of 21-40 yrs, six patients presented with stage II disease, whereas 12 patients presented with stage III disease. Of the 27 patients in the age group of 41-60 yrs, 11 presented with stage II and 16 presented with stage III. Of the five patients in the age group of 61-80yrs, 3 presented with stage IIB, and 2 presented with stage IIIB. Similar to clinical-stage patients of younger age groups are presenting with the advanced pathological stage. The majority (33f50) of the patients were presented with $\mathrm{pN} 1$ status, 12 with $\mathrm{pN} 0$ status, and 5 with pN2 stage.

\section{Correlation between Clinical and Pathological Staging:}

Clinically node-negative cases were $(20 f 50)$ but, pathologically node- negative cases were (12f50) which indicates that eight patients who were clinically negative for nodes found to be positive in the pathological examination. Clinical N1 was 25, but pathological N1 was 33. Clinically and pathologically, N2 was 5 .

In this study, when a correlation was made between the clinical stage and pathological stage, it was observed that out of 11 patients with clinical stage IIA 7 belonged to pathological stage IIA and 4 belonged to pathological stage IIB. Among 13 patients with clinical stage IIB, one patient was down staged to pathological stage IIA, eight patients belonged to pathological stage IIB, and 4patients were upstaged to pathological stage $111 \mathrm{~A}$. All patients with clinical stage IIIA remained in IIIA, and all patients with clinical stage IIIB also remained in pathological stage IIIB. It is observed that this change in the clinical stage to the pathological stage is due to a change in nodal status.

\section{Hormonal Receptor Status and Histological Type of Tumour}

In the present study, out of 50 patients, 36 cases were reactive for hormone receptors but negative for Her2neu. Eleven cases were triple-negative. Three cases were nonreactive for hormone receptors but positive for Her2neu. Triple positive cases are not present in this study.

Of the 50 patients 47 were infiltrating duct cell carcinoma, 2 were metaplastic carcinoma, and 1 is papillary carcinoma.70\% of infiltrating ductal cell carcinoma NOS type belong to lumina' type A. Of the 47 patients with infiltrating duct cell carcinoma 8 are triple-negative, 36 are ER, PR positive with Her 2 neu negative and only Her2neu positive type is 3 . In metaplastic and papillary carcinoma, triple negative cases were observed.

\section{Discussion}

Breast cancer is the most common cancer in females, both in developed and developing countries and is second common next only to lung cancer in bothsexes $^{(1,2)}$. In present study, all the patients were evaluated through proper history and complete physical examination, including various biochemical, pathological, and radiological investigations.

Following the evaluation, the diagnosis was confirmed, and patients were operated, and specimens sent for histopathological examination and assessment of hormone receptor status. The information obtained from the pathological studies is utilized to identify the pathological staging, and an attempt was made to correlate the clinical stage and pathological stage.

In this study, the mean age of breast cancer was $55 \mathrm{yrs}$. The youngest patient was $30 \mathrm{yrs}$ old, and 'oldest patient was $80 \mathrm{yrs}$ old. The incidence of carcinoma breast was found to be highest in the age group 41-60yrs (54\%). However, the incidence is rising in the younger population. In this study, patients with a young age have presented with advanced clinical and pathological stage

This observation can be compared to similar study in 
which Neelesh et al. ${ }^{3}$ found that mean age group was $45.2 \mathrm{yrs}$ and peak incidence was in the age group 41-60yrs, Okoiba $\mathrm{MN}$ et al. ${ }^{4}$ reported mean age as 38yrs, Aslam HM reported mean age as 45.66yrs, Samina et al. reported mean age as 47yrs and Mudgal reported mean age as 47yrs. In Asia, breast cancer incidence peaks among women in their forties, whereas in the United States and Europe, it peaks among women in their sixties.

In the present study, $100 \%$ of patients presented with a lump of varying duration; $20 \%$ of patients had a painful lump. Lumps in the breast are often ignored, and patients seek advice when the mass either increase in size suddenly or has become painful due to massage, hot fomentation, and hemorrhage, superseding infection, subsequent changes in the lump. According to Okoiba $\mathrm{MN}$ et al. ${ }^{(4)}$, most of the tumors were painless, and patients were likely to disregard these lesions. Reports from our hospital too suggest that low socioeconomic status, poverty, and ignorance are the major factors responsible for this.

In this study, the right breast is involved in $52 \%$ of cases. This finding can be compared to Neelesh et al. (3), in which the right breast is involved in $55.7 \%$ of cases. The involvement of the right or left breast varies with study to study. The preponderance of carcinoma of the right breast to the left breast is just a variation within standard limit with a small sample size, which needs further long term study. In the present study, the upper and outer quadrant was most commonly involved.

In the present series, the majority (40\%) of the patients in this study were presented with T2 tumors, 15 (30\%) presented with T3

tumors, and 15 (30\%) presented with T4 tumors. TATA MEMORIAL CANCER HOSPITAL registry reported majority $(60 \%)$ of patients with T2 lesions, S.B. Desai et al. also reported the same In present study clinical node- negative cases were $20(40 \%)$, and node-positive cases were $30(60 \%)$.

In the present study, most of the patients of carcinoma breast presented in stage III (52\%) followed by stage II (48\%). Stage I and stage IV disease were not encountered during the period of study. In Neelesh et al. ${ }^{(3)}$ study most of the patients of ca breast presented in stage II $(45.7 \%)$ followed by stage III $(32.8 \%)$ and stage IV $(17.1 \%)$, while only three patients $(4.2 \%)$ presented with stage I disease.

This difference in the stage distribution of breast cancer patients in the developing and developed countries has been largely attributed to lack of screening facilities, delay in seeking medical attention, poor socioeconomic status, poor health care systems, inadequate diagnostic and therapeutic facilities in developing countries.

In the present study, ER, PR hormone receptor positivity with Her2neu positivity was not encountered. 36 out of 50 patients $(72 \%)$ presented as luminal type A, 11 patients $(22 \%)$ presented as triple negatives, and three patients $(6 \%)$ presented as Her2neu positive.

Triple-negative cases were more common in clinical stage III and pathological stage III.

This data showed similarity with Adedayo et $\mathrm{al}^{(5)}$, which was done on 1134 carcinoma breast patients with a median age of $62.7 \mathrm{yrs}$ in Marshfield clinic $f$ St, Joseph hospital which reported ER $f \mathrm{PR}+\mathrm{Her} 2 \mathrm{neu}+$ as $10.2 \%$, ER $f \mathrm{PR}+\mathrm{Her} 2 \mathrm{neu}-$ as $68.9 \%$,

ER $f$ PR- Her2neu+ as $7.5 \%$ and triple-negative as $13.4 \%$. Out of 37 cases of infiltrating ductal cell carcinoma, $70 \%$ are luminal type $\mathrm{A}$, and $21.6 \%$ are triple-negative.

All cases in this study were offered surgery, and Modified Radical Mastectomy was done in all cases.BCS was not done inany patient as most of the patients come from rural areas, and as such, there was a chance of loss of follow up for chemoradiotherapy. Out of 50 patients, 18 patients $(36 \%)$ received neoadjuvant chemotherapy, which led to alleviate clinical symptoms and significant regression in tumor size, and that was followed by Modified Radical Mastectomy.

\section{Conclusion}

Due to illiteracy, low socioeconomic status, lack of awareness of the disease, and hesitancy to report to a doctor, most patients often present in late stages.

As in this study, there is the rising incidence of 
carcinoma breast in the younger population and higher incidence of disease in the age group of 41-60 years, all women above the age of 20 years should undergo clinical breast examination once in 3 years and above the age of 40 yrs annually.

All women above the age of 40 years should undergo screening mammography annually. Triple- negative cases were more common in patients with the advanced clinical and pathological stage. ER, PR, and Her2 neu receptor status assessment is recommended in all patients with breast cancer to facilitate neoadjuvant and adjuvant therapy and for assessing prognosis.

\section{References}

1. Bailey and love. Short practice of surgery. In Norman S. Williams, Christopher J.K. Bulstrode, P. Ronan O'Connell, editors. London: Hodder Arnold, $26^{\text {th }}$ edition, 2017.

2. Sabiston Textbook of Surgery, The biological basis of modern Surgical practice, In Courtney M. Townsend Jr. Beauchamp RD, Evers BM, Mattox KL, editors. Philadelphia: Saunders, 20 ${ }^{\text {th }}$ edition, Volume II, 2017.

3. Neelesh et al., International Journal of Surgery in May2016.

4. Okoiba MN, Osime U. Clinic Pathological study of carcinoma breast in Benirr city, Department of Surgery, College of Medical Science, University of Benin teaching hospitals Benin city Nigeria. African J Reprod Health.2001.

5. Breast cancer subtypes based on ER $f \mathrm{PR}$ and Her2 expression comparison of clinicopathological features and survival Adedayo A.ontilo, MD, MSCR, FACP; Jessica M.Engel, MSN. FNP - BC, Rober T. Greenlee. Ph.D.; and Bickol N. Mukesh, Ph.D. Clinical Medicine, and research Volume. 